\title{
Capacitação de profissionais para intervenção em comportamentos aberrantes: Elaboração de material didático e validação social
}

Thais Yazawa

Silvia Aparecida Fornazari

\section{RESUMO}

Pessoas com deficiência intelectual severa, muitas vezes, continuam à margem dos serviços de atendimento especializado, apesar das políticas de inclusão escolar e social para essas pessoas. Esses indivíduos podem apresentar comportamentos aberrantes: um conjunto de comportamentos mal adaptativos que podem ser fonte de estigmatização, dificultando a inclusão e o acesso ao tratamento especializado. 0 presente artigo pretendeu elaborar e validar socialmente um material didático para profissionais da saúde, utilizando os princípios básicos da Análise do Comportamento, para diminuição da apresentação dos comportamentos aberrantes e aumento da frequência de comportamentos adequados ao contexto de atendimento de pessoas com deficiência intelectual severa. 0 material didático foi entregue a 11 profissionais da saúde participantes, que realizaram as atividades propostas por ele. Na sequência, foi aplicado o Therapy Attitude Inventory (TAI) adaptado para realizar uma validação social do material desenvolvido, no qual $80 \%$ dos participantes avaliaram o material como acima da média. Tais resultados também puderam ser demonstrados na correlação do TAI e na entrevista final. Os participantes avaliaram que o material os auxiliou a compreender o comportamento e a intervir sobre ele de maneira adequada.

Palavras-chave: comportamento aberrante; material didático; análise do comportamento; educação especial.

\section{ABSTRACT}

\section{Professional training for intervention in aberrant behavior: Development of} teaching materials and social validation

People with severe intellectual disabilities are usually overlooked in what concerns specific care services despite the policies of school and social inclusion aimed at them. These individuals may present aberrant behaviors, a set of maladaptive behaviors which is likely to stigmatize and put these people aside from society, then complicating inclusion and specific treatment. Thus, the present paper aimed to develop and socially validate an instruction material by using basic principles of Behavior Analysis in order to decrease the display of aberrant behaviors and increase the frequency of behaviors appropriate to the context of health professionals who provide treatment for people with severe intellectual disabilities. The instruction material was addressed to 11 health professionals, who performed the activities proposed therein. Then, the participants were applied the Therapy Attitude Inventory (TAI), adapted in order to foster the social validation of the material, in which $80 \%$ of the participants appraised the material as above average. These results were also shown in TAl's correlation and in the final interview. Participants assessed that the material helped them understand the behavior and appropriately intervene in it.

Keywords: aberrant behaviors; instruction material; behavior analysis; special education.

A marginalização das pessoas com deficiência intelectual severa é um processo estrutural antigo em nossa sociedade, deixando-as à mercê de atitudes preconceituosas e afastadas de atendimentos especializados. Por vezes, são tratadas sem respeito e, se atendidas, o são de forma precária (Maciel, 2000). Um dos motivos para tal processo é a frequência dos

\section{Sobre os Autores}

T. Y. orcid.org/000-0001-8229-0459 Universidade Estadual Paulista Júlio de Mesquita Filho (UNESP)

- Bauru, SP

tatayazawa@gmail.com

S. A. F.

orcid.org/0000-0001-6012-5911 Universidade Estadual Paulista Júlio de Mesquita Filho (UNESP) - Bauru, SP

silfornazari@gmail.com

\section{Direitos Autorais}

Este é um artigo de acesso aberto e pode ser reproduzido livremente, distribuído,

transmitido ou modificado, por qualquer pessoa desde que usado sem fins comerciais. 0 trabalho é disponibilizado sob a licença Creative Commons CCBY-NC.

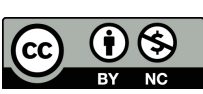


comportamentos chamados "aberrantes". Tais comportamentos seriam os comportamentos agressivos (heteroagressão e autoagressão), comportamentos disruptivos como chutar paredes, cadeiras, bater em objetos, derrubar objetos propositalmente, arremessar objetos, entre outros. Ainda são considerados aberrantes comportamentos como comer objetos não comestíveis, como brinquedos, peças de roupas e cabelos, e/ou masturbar-se em público. Quando tais comportamentos são apresentados pelo indivíduo em alta frequência e por muito tempo, tendem a se tornar "cristalizados", ou seja, de difícil modificação, e a causar danos físicos e sociais, na maioria das vezes considerados graves (Fornazari, 2000, 2005).

Além da falta de estrutura necessária, o atendimento de saúde pode ser um desafio para os profissionais (Leonardo, Bray, \& Rossato, 2009; Maciel, 2000). Isso deve-se ao fato de que alguns indivíduos com deficiência intelectual destacamse pela frequência e estranheza de certos comportamentos que apresentam, como autolesão, agressão a outros, comportamentos estereotipados ou crises comportamentais (surtos e birras), os quais são as reações consideradas "aberrantes" (Mendes, 2001). Gomes e Barbosa (2006) constataram a falta de preparo e de informações dos professores de ensino fundamental, com relação ao manejo, metodologias de ensino e práticas educacionais. Tal despreparo estende-se aos profissionais de saúde e não há bases na formação em saúde que os habilite para tal função (Höher \& Dotto, 2006; Maciel, 2000).

De acordo com Fornazari (2005), conhecimento a respeito dos princípios da Análise do Comportamento pode auxiliar esses profissionais e não devem estar restritos apenas ao psicólogo. Muitas vezes, professores ou profissionais que atendem essa população não dispõem de métodos que consigam modificar comportamentos inadequados e/ou aberrantes, reduzindo-os e aumentando a frequência de comportamentos adequados ao contexto.

Capacitações em Análise do Comportamento para professores do ensino fundamental e universitário vêm sendo realizadas com resultados positivos, como no estudo de Fornazari, Kienen, Tadayozzi, Ribeiro, e Rossetto (2012), que, por meio de ensino informatizado, capacitaram quatro professoras de uma escola municipal a utilizarem princípios da Análise do Comportamento e o procedimento de Reforçamento Diferencial de Comportamento Alternativo (DRA). Ausec e Fornazari (2014) utilizaram o software ENSINO para ensino de professores universitários, com novos arranjos de contingências como alteração da linguagem e apresentação do conteúdo. Tais estudos podem contribuir para o processo de inclusão, possibilitando que os conhecimentos produzidos pela Análise do Comportamento sejam estendidos para outras áreas de conhecimento. Os participantes dos estudos citados, após realizarem as capacitações, foram entrevistados, preencheram um questionário de validação social e realizaram pós-teste, todos com um aumento no acerto das questões sobre princípios da Análise do Comportamento e DRA quando comparados com o pré-teste.

Procedimentos de reforçamento diferencial estão entre as técnicas mais utilizadas para diminuir apresentação de comportamentos indesejáveis, envolvendo reforçamento positivo (Friman, Barnard, Altman \& Wolf, 1986; Lennox, Miltenberger, Spengles \& Erfanian, 1988). São procedimentos que visam aumentar a frequência de emissão de comportamentos adequados, melhorando o repertório comportamental do indivíduo. O reforçamento diferencial de comportamento alternativo (DRA) é um dos mais utilizados, de acordo com Petscher, Rey, \& Bailey (2009), assim como o reforçamento diferencial de comportamento incompatível (DRI) tem sua importância para redução de comportamentosproblema (O'Brien \& Repp, 1990). O DRA ou Differential reinforcement of alternative behavior, regularmente utilizado na análise do comportamento aplicada para reduzir respostas de agressão e autoagressão, é um procedimento que envolve o ensino de um comportamento equivalente e apropriado, com a mesma função do comportamento inapropriado (Liddon, Kelley, \& Podlesnik, 2017). O DRI ou Differential reinforcement of incompatible behavior, consiste na apresentação de estímulos reforçadores positivos contingentemente à ocorrência de um ou mais comportamentos topograficamente incompatíveis com o comportamento aberrante (Miltenberger, 2001). 0 reforçamento diferencial de outros comportamentos (DRO ou Differential reinforcement of other behaviors) é a apresentação contingente de estímulos reforçadores na ausência do comportamento-alvo (Conyers, Miltenberger, Romaniuk, Kopp, \& Himle, 2003).

Estudos publicados nos Estados Unidos avaliaram formas mais eficientes de procedimentos aplicados para diferentes níveis de comprometimento intelectual dos participantes (Friman et al., 1986; Piazza, Moes, \& Fisher, 1996; Roane, Fisher, Sgro, Falcomata, \& Pabico, 2004). Com base nesses estudos, tais procedimentos podem ser aplicados em pacientes de profissionais que fazem atendimento em saúde, possibilitando que estes possam desempenhar as atividades para as quais foram qualificados. No estudo de Friman e colaboradores (1986), por exemplo, os autores treinaram um professor e uma mãe para serem capazes de realizar DRA, DRO e DRI em uma criança com comportamentos aberrantes, diminuindo a ocorrência desses comportamentos e ensinando novos comportamentos adequados, possibilitando ganhos sociais. Este estudo serve de apoio para iniciativas de 


\section{W'INTERACÃO EM ¿Y. PSICOLOGIA}

capacitação para profissionais da saúde, da educação e outras esferas que atendam às pessoas com deficiência intelectual.

Considerando os aspectos especificados, construir um material de capacitação para profissionais consiste em um objetivo relevante. Todas as pessoas merecem um atendimento tanto em saúde quanto em educação, realizado de maneira especializada e competente; e há uma lacuna nesse sentido, quando o público-alvo são as pessoas com deficiência. A Análise do Comportamento desenvolveu técnicas e estratégias de ensino personalizado, que podem atender esses profissionais. Keller (1968) criou o Personalized System of Instruction (Eyre, 2007), que possibilita um aprendizado que considera o ritmo individual, com o conteúdo dividido em blocos, com gradação de dificuldade e sem a presença do professor. Nesse procedimento, há uma equipe acadêmica que proporciona suporte ao aprendiz e avaliações após completado o bloco de conteúdo. O programa de Keller pode ser adaptado em versões informatizadas ou de texto programado, como os estudos de Couto (2009), Eyre, Crone-Todd, Jones, Hutchens e Pear (2005).

Nesse sentido, Hamada (2014) desenvolveu um material didático autoinstrucional direcionado a pais de pessoas que emitem comportamentos aberrantes, em que a autora observou que, apesar de a aprendizagem ser complexa, por apresentar conceitos específicos da Análise do Comportamento e exigir grau de escolaridade, os familiares apresentaram satisfação em participar da pesquisa, do método de intervenção e dos procedimentos abordados. 0 material desenvolvido consiste em uma apostila autoinstrucional de 140 páginas, com textos explicativos e ilustrações de situações em que o comportamento aberrante era apresentado pelos filhos e quais seriam os comportamentos esperados dos pais, de acordo com o DRA e DRI. Após cada capítulo, exercícios eram propostos, com feedback imediato para o aprendiz acompanhar seu desempenho.

A partir desse estudo, observou-se a necessidade da elaboração de um material didático destinado aos profissionais da saúde, fundamentado nos princípios da Análise do Comportamento, oferecendo conhecimentos de intervenções para melhorar os atendimentos às pessoas que emitem comportamentos aberrantes. Tal necessidade foi observada pelo relato dos próprios pais, sobre a necessidade de todos terem acesso a esse tipo de conhecimento, para prover capacitação contínua a todos os níveis que atendem às crianças com deficiência intelectual. A capacidade de intervir em comportamentos aberrantes apresentados por seus pacientes pode contribuir para que o trabalho do profissional de saúde possa ser realizado com eficácia dentro de sua especialidade. Trabalhar com eficácia seria realizar os procedimentos de forma correta, utilizando de forma correta, os recursos disponíveis (Suárez, 2000), o que otimizaria o trabalho realizado pelos profissionais da saúde.

O objetivo do presente estudo foi elaborar, aplicar e validar socialmente um material didático, utilizando os princípios da Análise do Comportamento para a diminuição de comportamentos aberrantes e aumento da frequência de comportamentos adequados ao contexto de atendimento para profissionais que trabalham com pessoas com deficiência intelectual severa. A validação social, como instrumento científico, é importante para verificar, dentro da comunidade que foi público-alvo da pesquisa, sua viabilidade, aplicabilidade e compreensão, com objetivo de averiguar a satisfação do participante. Implica em analisar pelo menos três níveis: 1) a significância social dos objetivos; 2) a aceitabilidade social dos procedimentos de intervenção; e 3) a importância social dos efeitos - a satisfação dos participantes da pesquisa (Wolf, 1978).

Para elaboração do material didático foram considerados "Os cinco princípios básicos da Programação de Ensino" (Botomé, 1970), sendo estes: 1) pequenos passos: que se refere à necessidade de adotar pequenas unidades de ensino de fácil aprendizagem e, aos poucos, aumentar a dificuldade, para uma aprendizagem sem erros; 2) resposta ativa: refere-se à necessidade do aluno de participar da aprendizagem, exteriorizando seus comportamentos para desenvolver as habilidades; 3) verificação imediata: diz respeito à importância de verificar imediatamente seu desempenho, para aprender melhor; 4) ritmo individual: explana a importância de cada um aprender em seu ritmo; 5) teste de avaliação: o último princípio, tem relação com a importância de um exame sobre o que cada aluno faz em cada unidade de ensino, avaliando, revisando e aperfeiçoando o programa sempre que necessário. Tais princípios são semelhantes aos dos estudos de Keller (1968), com o Personalized System of Instruction (PSI). Para o presente estudo foi utilizada a compreensão de "unidades de ensino" como pequenos trechos de conteúdos explicativos, com exercícios seguidos de feedback.

\section{MÉTODO}

\section{PARTICIPANTES}

Participaram deste estudo 11 profissionais (nove mulheres e dois homens, com idades entre 24 e 35 anos), empregados em instituições especializadas no atendimento às pessoas com deficiência intelectual, com formação 


\section{H: INTERACÃO EM LAS PSICOLOGIA}

superior em áreas da saúde. Eram seis fisioterapeutas, dois fonoaudiólogos e três terapeutas ocupacionais, todos com pelo menos um curso de especialização. 0 participante com menos tempo de instituição estava empregado há oito meses, e o empregado há mais tempo, 14 anos. 0 critério para participação no estudo foi trabalhar com pessoas que apresentassem comportamentos aberrantes por um tempo mínimo de seis meses.

\section{INSTRUMENTOS}

Foram utilizados para o presente estudo uma adaptação do Therapy Attitude Inventory (TAI) de Eyberg (1993) e um roteiro de entrevista semiestruturado. O Inventário TAI de Eyberg (1993) consiste em um questionário com 10 questões com respostas de múltipla escolha de um a cinco, para avaliar a satisfação ao impacto de treino de pais em áreas como disciplina, qualidade da interação pai-filhos, comportamento dos filhos e outras interações familiares. Para o presente estudo foram reformuladas oito questões, direcionando algumas delas para a percepção dos profissionais em relação aos comportamentos apresentados pelos pacientes e a percepção de melhora/piora do próprio participante em relação aos conceitos e procedimentos ensinados. Os temas abordados pelas questões tangiam as seguintes áreas: técnicas, aprendizagem, relacionamento, confiança, percepção, esclarecimento, material didático e pesquisa, por serem as áreas de interesse para a presente pesquisa. As respostas são escalonadas de 1 a 5 , sendo a resposta 1 a que se refere à maior insatisfação, e a resposta 5, à maior satisfação. Foi incluída uma questão aberta na qual o participante poderia deixar sugestões, dúvidas e observações.

O texto das questões foram:

I. Considerando as técnicas para lidar com o comportamento aberrante do(a) meu(minha) paciente, eu penso que eu aprendi

II. Considerando a forma de ensinar novos comportamentos a meu(minha) paciente, eu sinto que o que eu aprendi

III. Considerando o relacionamento entre mim e meu(minha) paciente, eu sinto que nós lidamos

IV. Eu sinto, neste momento, que o controle que tenho sob os comportamentos aberrantes de meu(minha) paciente está

V. Neste momento, eu percebo que os comportamentos aberrantes apresentados pelo meu(minha) paciente estão

VI. Em que grau a leitura do material tem ajudado com outros problemas gerais, pessoais ou familiares não diretamente relacionados ao seu(sua) paciente?

VII. Eu sinto que o tipo de material que foi usado para me ajudar a reduzir os comportamentos aberrantes do(a) meu(minha) paciente foi

VIII. Meu sentimento geral sobre a pesquisa que eu participei é

0 roteiro de entrevista semiestruturada era composto por 16 questões, que podem ser visualizadas na Tabela 1:

\section{Tabela 1. Questionário semiestruturado}

\begin{tabular}{l} 
Perguntas \\
\hline Idade \\
Sexo \\
Formação \\
Ocupação \\
Tempo que trabalha na instituição \\
A leitura do material didático te ajudou a compreender os \\
comportamentos aberrantes e a intervir sobre eles de \\
maneira adequada? Se sim, como? Se não, por quê? \\
Você já leu materiais parecidos com esse? Quais? \\
Você já tinha ouvido falar em "comportamentos \\
aberrantes"? \\
Se SIM: O que você entendia por "comportamentos \\
aberrantes"? Se NÃO: O que você pensaria sobre o que \\
são comportamentos aberrantes, antes de conhecer o \\
material? \\
Considerando o que você aprendeu, o que são \\
"comportamentos aberrantes"? \\
O que achou do termo "comportamentos aberrantes"? \\
O que achou mais interessante no material? \\
Você conseguiu agir com seu paciente do modo como o \\
material ensina? Se SIM, como foi essa experiência? Se \\
NÃO, qual foi o motivo? \\
Com relação à pergunta 5 do questionário, sobre a \\
condição do comportamento do(a) seu(sua) paciente(a) \\
atualmente, explique por que você acha que piorou/não \\
mudou/melhorou \\
Você acha que futuramente, aplicando mais vezes e por \\
mais tempo os procedimentos ensinados no material, \\
você terá resultados melhores? Explique \\
Quais sugestões tem para melhorar o material? \\
\hline
\end{tabular}

Os conceitos incluídos no material foram baseados nos estudos de Mello (2012), Martins (2013), Ausec (2013), Ausec e Fornazari (2014) e Bassetto (2015) que utilizaram o software ENSINO (Fornazari, 2011), desenvolvido para o ensino de princípios e procedimentos de Análise do Comportamento. Em relação aos procedimentos estudados, Mello (2012), Martins (2013), Ausec (2013), Ausec e Fornazari (2014) e Bassetto (2015) utilizaram a Análise Funcional e os procedimentos de DRA. Hamada (2014), além 
dos procedimentos utilizados pelos estudos mencionados, incluiu o ensino do procedimento de DRI, que pode ser bastante útil na intervenção com pessoas que apresentam comportamentos aberrantes, especialmente para comportamentos de agressão e autoagressão. Os conceitos utilizados pelos autores são os conceitos de comportamentos, reforço, contingência, extinção, discriminação, generalização, análise funcional e esquemas de reforçamento diferenciais de comportamentos alternativos e incompatíveis. A estrutura do material é apresentada na Tabela 2.
O material desenvolvido é composto por 110 exercícios, 100 de múltipla escolha e 10 dissertativos, divididos em 15 capítulos. A primeira parte do material é composta por princípios básicos da Análise do Comportamento, a segunda parte aborda o procedimento de Análise funcional, e a terceira parte é composta por exercícios de DRA e DRI ilustrados e contextualizados. Os capítulos contêm textos explicativos, ilustrações, exercícios e gabarito após os exercícios. A dificuldade da execução do material foi aumentando de forma gradual, dos conceitos mais simples aos mais complexos.

\section{Tabela 2. Capítulos e descrição do material}

\begin{tabular}{|c|c|}
\hline Capítulo & Descriçāo do capítulo \\
\hline $\begin{array}{l}1 \text { - O que é antecedente, resposta e consequência? } \\
\text { Aprendendo os componentes do comportamento }\end{array}$ & Definiçōes de antecedente, resposta e consequência \\
\hline 2 - Contingência & Definição do conceito de contingência \\
\hline 3 - Punição: por que essa palavra tão feia? & Definição do conceito de punição \\
\hline 4 - Extinçāo: o que é? & Definiçāo do conceito de extinçāo \\
\hline $\begin{array}{l}5 \text { - Discriminaçāo e Generalizaçāo: dois conceitos de } \\
\text { aprendizagem }\end{array}$ & Definiçāo dos conceitos de discriminaçāo e generalizaçāo \\
\hline 6 - Reforço! Um grande aliado & Definiçāo do conceito de reforço \\
\hline 7 - Reforçando os conceitos aprendidos & Exercícios para fixaçāo dos conceitos aprendidos \\
\hline $\begin{array}{l}8 \text { - Análise Funcional: estudamos tudo isso para } \\
\text { chegarmos aqui! }\end{array}$ & $\begin{array}{l}\text { Definição do conceito de Análise funcional - explicação de } \\
\text { como realizá-la, exemplos e ilustraçōes }\end{array}$ \\
\hline 9 - Registro de respostas & $\begin{array}{l}\text { Como registrar os comportamentos observados - com } \\
\text { modelos explicativos }\end{array}$ \\
\hline 10 - Realizando o registro dos consequentes & $\begin{array}{l}\text { Como registrar os comportamentos observados - com } \\
\text { modelos explicativos } \\
\text { Seis exercícios }\end{array}$ \\
\hline 11 - Fazendo o registro dos antecedentes & $\begin{array}{l}\text { Como registrar os comportamentos observados - com } \\
\text { modelos explicativos }\end{array}$ \\
\hline $\begin{array}{l}12 \text { - Funçōes do comportamento e análise funcional a } \\
\text { partir da folha de registros }\end{array}$ & $\begin{array}{l}\text { Definição e conceituação das funçōes comportamentais, } \\
\text { com exemplos e ilustraçōes }\end{array}$ \\
\hline $\begin{array}{l}13 \text { - Instalando um novo comportamento usando } \\
\text { Intervençāo por meio de Esquemas de Reforçamento - } \\
\text { DRI }\end{array}$ & $\begin{array}{l}\text { Definiçāo e conceituação dos esquemas de reforçamento, } \\
\text { exemplos e ilustraçōes }\end{array}$ \\
\hline $\begin{array}{l}14 \text { - Procedimento de reforçamento diferencial de } \\
\text { comportamentos alternativos - DRA }\end{array}$ & $\begin{array}{l}\text { Definiçāo e conceituaçāo dos esquemas de reforçamento, } \\
\text { exemplos e ilustraçōes }\end{array}$ \\
\hline 15 - Exercícios para fixar os Procedimentos & 35 exercícios comentados e contextualizados \\
\hline
\end{tabular}

Todo o material foi elaborado para Yazawa (2015), utilizando os conceitos do software ENSINO (Fornazari, 2011) e explicações e conceitos do livro Moreira e Medeiros (2007). Cada capítulo iniciava com uma breve descrição do conceito, com exemplos do cotidiano para facilitar a compreensão. Posteriormente era apresentada uma ilustração (desenvolvida por Leandro Toledo), com o objetivo de ilustrar o conceito. Para definir a dificuldade dos capítulos desenvolvidos, foi utilizada a decomposição das classes de comportamentos esperados para atingir o próximo nível de aprendizado. Classes de comportamento não se referem a um comportamento determinado, mas a comportamentos que apresentam a mesma função, formando uma classe (Kienen, 2008), ou seja, para compreender o que é contingência, o participante primeiro precisa compreender os conceitos de antecedente, resposta e consequência, por exemplo. 


\section{PROCEDIMENTOS}

Procedimentos éticos. O estudo foi aprovado por Comitê de Ética (CAEE 42860315.2.0000.5231). Após contato com as instituições de atendimento de saúde para crianças com deficiência intelectual, foram marcados os encontros com os participantes. No primeiro encontro, estes foram informados sobre o estudo e foram convidados a participar deste. Os profissionais assinaram o Termo de Consentimento Livre e Esclarecido e, para a entrevista final, todos aceitaram a gravação de seus relatos com a finalidade de transcrição.

Disponibilização do material didático. Os profissionais receberam o material em um encontro individual ou em pequenos grupos. Após esclarecimentos sobre o material e o projeto, foi entregue 0 material com as explicações necessárias para resolução das atividades propostas, e dado o prazo de um mês, aproximadamente, para entrega e entrevista.

Encontro para devolução do material didático. Após a leitura do material didático, foi agendado outro encontro com os participantes da pesquisa, devolvido o material e aplicado o TAl adaptado para realizar a validação social quanto à aceitabilidade dos procedimentos propostos pelo material. Ao final, foi realizada uma entrevista para levantar aspectos relevantes com relação ao preenchimento do material, dificuldades, pontos fortes e sugestões. As entrevistas foram individuais e realizadas entre atendimentos dos profissionais, em horário de trabalho, com duração máxima de 10 minutos.

Procedimentos de análise dos dados coletados. Foram coletados três tipos de dados, sendo esses: as respostas às atividades propostas pelo material didático preenchidas pelo participante, as respostas do questionário TAl adaptado e a entrevista realizada pela pesquisadora.

a) Material didático

Para o estudo, foram considerados os materiais preenchidos em pelo menos 75 questões $(80 \%$ das atividades). Foram tabuladas as respostas corretas, parcialmente corretas, incorretas e exercícios em branco. Para exercícios parcialmente corretos, foi considerado que o participante tenha acertado a maior parte da resposta. Para respostas incorretas, foram consideradas respostas que não se assemelhavam às respostas sugeridas no gabarito. Foi considerada apenas a primeira resposta do participante, observando que alguns rasuraram os exercícios, por correção realizada por eles mesmos por meio do gabarito oferecido. Essas informações foram organizadas e tabuladas.

0 material foi avaliado quanto à quantidade de acertos dos participantes. Somaram-se todos os acertos, todas as respostas incorretas, exercícios parcialmente corretos e exercícios em branco, de todos os participantes, divididos por capítulos.

\section{b) Inventário de Satisfação (TAl) adaptado}

A validação social foi realizada pelo instrumento TAI adaptado respondido pelos participantes. A análise foi feita considerando o número de respostas dentro da escala dada pelo instrumento para cada questão.

\section{c) Entrevista}

Os resultados foram analisados de acordo com o roteiro de entrevista semiestruturada e comparados com os resultados do TAl adaptado em suas semelhanças e diferenças no relato sobre os tópicos que atendiam os mesmos assuntos. Para oferecer uma análise qualitativa, sistematizando as respostas com as mesmas funções e conteúdos e aglomerando por tópicos sobre as opiniões.

\section{RESULTADOS E DISCUSSÃO}

\section{Avaliação do material didático}

Os resultados quanto às respostas dos participantes aos exercícios propostos estão na Tabela 3 , na qual mostra-se que $92 \%$ dos participantes acertaram as questões da Primeira Unidade, que são referentes aos conceitos de princípios básicos em Análise do comportamento, 3,7\%

Tabela 3. Quantidade de respostas em cada categoria da Unidade 1 do material didático

\begin{tabular}{lcccc}
\hline \multicolumn{1}{c}{ Capítulos } & Correto & Parcialmente correto & Incorreto & Em branco \\
\hline Cap. 1. Componentes do comportamento, N=88 & $69(78 \%)$ & 0 & $4(5 \%)$ & $15(17 \%)$ \\
Cap. 2 Contingência, N=99 & $98(99 \%)$ & 0 & $1(1 \%)$ & 0 \\
Cap. 3 Puniçāo, N=66 & $57(86 \%)$ & 0 & $3(5 \%)$ & $6(9 \%)$ \\
Cap. 4 Extinção, N=55 & $50(92 \%)$ & 0 & $4(7 \%)$ & $1(1 \%)$ \\
Cap. 5 Discriminação e Generalização, N=88 & $86(98 \%)$ & 0 & $2(2 \%)$ & 0 \\
Cap. 6 Reforço, N=44 & $43(98 \%)$ & 0 & $1(2 \%)$ & 0 \\
Cap. 7 Lembrando (...), N=154 & $142(92 \%)$ & $2(1 \%)$ & $7(4,5 \%)$ & $3(2,5 \%)$ \\
Totais: 594 & $545(92 \%)$ & $2(0,33 \%)$ & $22(3,7 \%)$ & $25(4,2 \%)$ \\
\hline
\end{tabular}




\section{He MTERACíOEM ET. PSICOLOGIA}

erraram, 0,33\% acertaram parcialmente e 4,2\% deixaram questões em branco, no total de 54 exercícios propostos nessa unidade. $O$ " $\mathrm{N}$ " é a quantidade de respostas de todos os participantes da pesquisa. A quantidade de acertos indica que a Primeira Unidade está adequada ao ensino do conteúdo proposto. Os participantes apresentaram mais dificuldades, com mais erros, no Capítulo 4 - Extinção -, e menos no Capítulo 2 - Contingência -, que teve mais acertos.

Os dados apresentados na Tabela 3 estão relacionados aos conteúdos ministrados na Unidade 2. Após compreender os conceitos básicos da Análise do Comportamento (Unidade 1 ), os participantes entraram em contato com preceitos da Análise Funcional.

$\mathrm{Na}$ Tabela 4, é possível visualizar que a Unidade 2 foi a menor das unidades, com um menor índice de acertos (81\%), $2,72 \%$ de respostas parcialmente corretas, $4,54 \%$ de respostas incorretas e $12 \%$ de respostas em branco. Pode-se sugerir que o menor índice de acertos foi ocasionado pela configuração dos exercícios, sendo eles dissertativos, ou pelo grau de dificuldade de execução. Os exercícios dissertativos consistiam em ilustrações de situações em que o comportamento aberrante era emitido e pedia-se ao participante para escrever qual era a função do comportamento demonstrado, por meio da análise de estímulos antecedentes e consequentes. O objetivo era verificar se o participante conseguiria realizar a resposta correta sem nenhuma dica, o que pode ser um fator de dificuldade. Para o exercício a respeito de Análise funcional, foi estruturada uma tabela em branco, onde o participante deveria preencher com os antecedentes, a resposta e os consequentes. Apesar de ser o menor índice de acertos, pode ser considerada uma porcentagem alta de acertos (81\%), o que sugere aprendizado.

\section{Tabela 4. Quantidade de respostas em cada categoria na Segunda Unidade}

\begin{tabular}{lcccc}
\hline \multicolumn{1}{c}{ Capítulos } & Correto & Parcialmente correto & Incorreto & Em branco \\
\hline Cap. 8 - Conceito de Análise funcional, $\mathrm{N}=33$ & $29(87,8 \%)$ & $2(6,06 \%)$ & $2(6,06 \%)$ & 0 \\
Cap. 9 - Registro de respostas, $\mathrm{N}=33$ & $29(87,8 \%)$ & $2(6,06 \%)$ & $2(6,06 \%)$ & 0 \\
Cap. 12 - Funçōes do comportamento, $\mathrm{N}=44$ & $40(90,0 \%)$ & 0 & $3(6,81 \%)$ & $1(2,27 \%)$ \\
Totais: 110 & $89(81 \%)$ & $3(2,5 \%)$ & $5(4,5 \%)$ & $13(12 \%)$ \\
\hline
\end{tabular}

Na Tabela 5 estão os resultados dos participantes na última Unidade do livro (Terceira Unidade), com exercícios para compreensão dos conceitos e procedimentos, ilustrados e contextualizados.

A Terceira Unidade contemplou exercícios relacionados aos procedimentos de DRI e DRA e um capítulo com diversos exercícios situacionais para fixação do conhecimento, no total de 46 exercícios. A maioria dos participantes $(91,8 \%)$ acertou os exercícios, $6,5 \%$ erraram e $1,5 \%$ deixaram em branco. Não houve exercícios parcialmente corretos porque essa unidade foi constituída por questões objetivas. Os exercícios eram compostos por ilustrações e situações em que o comportamento aberrante era emitido e um procedimento de intervenção era necessário, com questões objetivas sobre cada caso.

Os exercícios foram incluídos no material, considerando as teorias de Keller (1968) e Botomé (1970), estruturados em pequenos passos, aumentando a dificuldade de forma gradual ao passar dos capítulos. O feedback imediato, também preconizado pelos autores, foi disponibilizado por meio de um gabarito comentado, fator esse que pôde alterar a confiabilidade das respostas, pois o participante podia copiar as respostas corretas. No estudo de Hamada (2014), no qual a Terceira Unidade foi estruturada em questões dissertativas, foi observado um número alto de exercícios em branco $(33,8 \%)$. Para o presente estudo, os exercícios foram revistos, com aumento no número de exercícios e no número de exercícios objetivos. Com tais alterações, apenas 1,58\%

Tabela 5. Quantidade de respostas em cada categoria na Unidade 3

\begin{tabular}{lcccc}
\hline \multicolumn{1}{c}{ Capítulos } & Correto & Parcialmente correto & Incorreto & Em branco \\
\hline Cap. 13 - DRI, N=66 & $57(86,36 \%)$ & - & $9(13,63 \%)$ & 0 \\
Cap. 14 - DRA, N=66 & $52(78,78 \%)$ & -- & $8(12,12 \%)$ & $6(9,09 \%)$ \\
Cap. 15 - Exercícios sobre reforçamento & $356(95,18 \%)$ & -- & $16(4,27 \%)$ & $2(2,13 \%)$ \\
diferencial, N=374 & $465(91,8 \%)$ & -- & $33(6,5 \%)$ & $8(1,5 \%)$ \\
Totais: 506 &
\end{tabular}


dos participantes não responderam aos exercícios dessa unidade.

\section{SATISFAÇÃO DOS PARTICIPANTES, AVALIADA POR MEIO DO TAI ADAPTADO}

Na Figura 1 são apresentados os resultados em relação ao Inventário de Satisfação adaptado para o presente estudo. Nenhum participante respondeu o nível 1 de satisfação, $1 \%$ respondeu o nível 2, 18\% responderam o nível 3, 61\% responderam o nível 4 e $19 \%$ responderam o nível 5 . A maioria dos participantes (80\%) avaliou o material como acima da média, respondendo os níveis 4 e 5 , demonstrando satisfação em participar da pesquisa. Os níveis de 1 a 5 podem ser identificados, de acordo com as perguntas: Técnicas - Nada (1), Quase nada (2), Poucos procedimentos úteis (3), Vários procedimentos úteis (4), Muitos procedimentos úteis (5); Aprendizagem - Atrapalhará (1), Adiantará pouco (2), Não fará diferença (3), Trará bons resultados (4), Solucionaria a maioria dos meus problemas (5); Relacionamento - Muito pior que antes (1), Um pouco pior que antes (2), 0 mesmo que antes (3), Um pouco melhor que antes (4), Muito melhor que antes (5); Confiança - Muito pior (1), Um pouco pior (2), O mesmo (3), Um pouco melhor (4), Muito melhor (5); Esclarecimento - Atrapalhou muito (1), Atrapalhou pouco (2), Nem ajudou, nem atrapalhou (3), Ajudou um pouco (4), Ajudou muito (5); Material didático -

Número de respostas no Inventário de Satisfação adaptado (TAI)

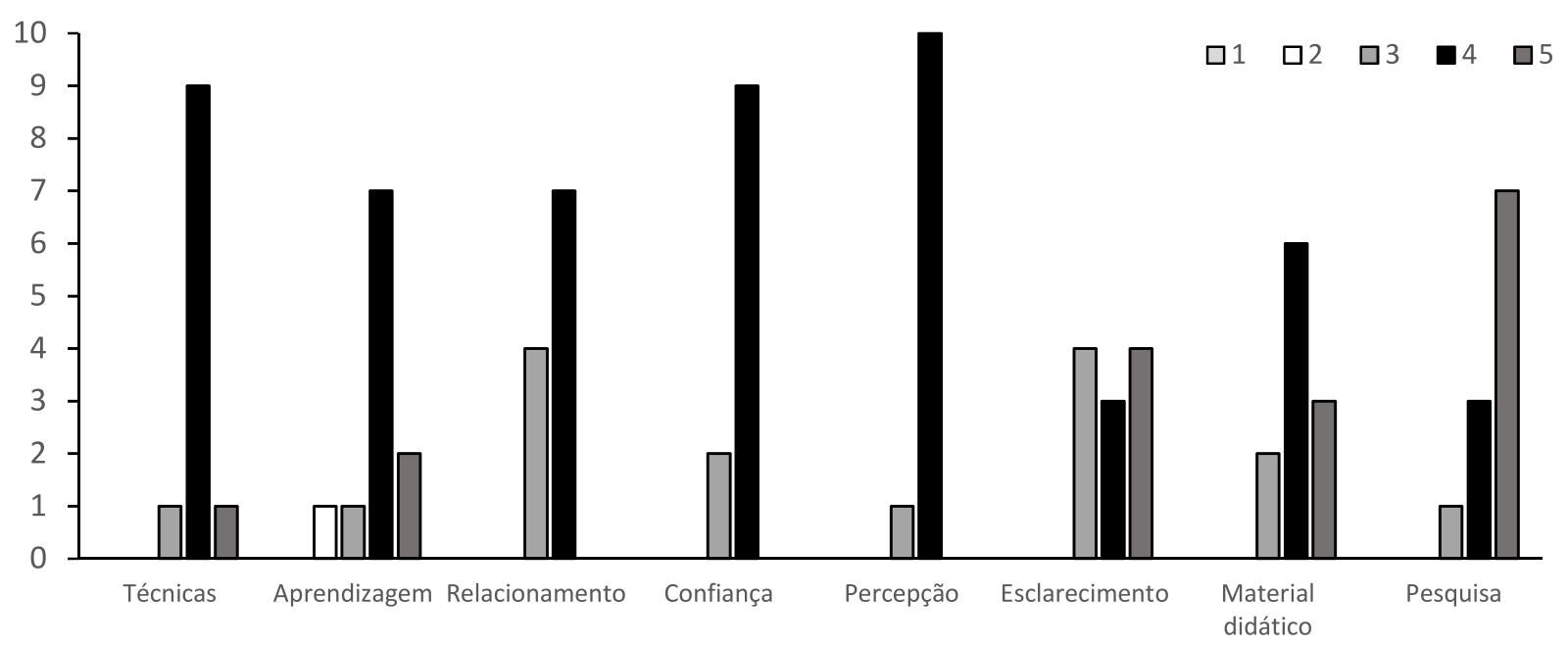

Figura 1. Número de respostas em cada categoria do Inventário de Satisfaçāo adaptado.

Muito fraco (1), Fraco (2), Adequado (3), Bom (4), Muito bom (5); Pesquisa - Detestei (1), Não gostei (2), Sinto-me neutro (3), Gostei um pouco (4), Gostei muito (5).

Pode-se sugerir que o material alcançou os objetivos da validação social, por ter sido avaliado acima da média pela maioria dos participantes (as categorias 4 e 5 do TAI), prestando esclarecimentos acerca dos comportamentos aberrantes, um fenômeno pouco discutido, segundo Maciel (2000) e Mendes (2001), provendo ferramentas para lidar com tais comportamentos. Os participantes mostraram satisfação em participar da pesquisa e avaliaram a intervenção como apropriada.

\section{RELATO VERBAL DOS PARTICIPANTES SOBRE O MATERIAL}

0 relato verbal dos participantes foi comparado com as respostas ao TAl sobre sua percepção de seu aprendizado, mudanças comportamentais nos pacientes e opinião sobre o material, com o objetivo de complementar os dados obtidos. Os resultados serão apresentados por categorias gerais de análise de conteúdo (Carlomagno \& Rocha, 2016).

\section{SOBRE A COMPREENSÃO DO COMPORTAMENTO}

Durante as entrevistas, apenas um participante relatou que o material o ajudou a compreender os comportamentos aberrantes, mas não a intervir:

Trabalhando em instituição, a gente tem hierarquia e a gente não pode resolver tudo sozinha. (P.1) 


\section{H'INTERACÃO EM ET PSICOLOGIA}

Dois participantes relataram que o material ajudou a compreender os comportamentos aberrantes e a intervir sobre eles:

Mostrando pra mim a forma que eu consigo lidar com o
paciente quando ele começa com esses
comportamentos, né (sic). (P.2)

Como entender o comportamento e aplicar o que foi ensinado, né. (P.6)

O relato verbal dos participantes pode ser compreendido em conjunto com a avaliação do TAl, demonstrando que materiais didáticos podem e devem ser utilizados em capacitações direcionadas à profissionais da saúde.

\section{MATERIAIS DIDÁTICOS}

Dois participantes já tiveram contato com materiais parecidos com o material desenvolvido, P.2 e P.7.

Já. Fiz no [nome da instituição de ensino], eu fiz o... fizemos pelo computador, né? (sic). (P.2)

A capacitação relatada por P.2 foi o estudo de Fornazari e colaboradores (2014), realizada em um instituto de trabalho com cegos, com o objetivo de capacitar os profissionais a aumentar o repertório de comportamentos adaptativos dos pacientes da instituição. A capacitação que trata este artigo pode ser entendida como mais uma intervenção com objetivo de melhorar as habilidades dos profissionais da saúde no trato dos problemas de comportamento, permitindo que os profissionais desempenhem suas atividades de forma adequada.

\section{OPINIÕES SOBRE O MATERIAL DIDÁTICO}

Referente ao que os participantes acharam mais interessante no material, as opiniões foram diversas, todos os participantes opinaram positivamente sobre o material.

Eu acho que a didática, os quadrinhos exemplificando ficaram bons. (P.4)

Os exemplos que eles davam pra (sic) gente entender como (sic) fazer, a dica que eles davam pra gente poder intervir no atendimento. (P.5)

[...] é essas, tipo a tarefinha, os exercícios. Os exercícios que fez assim, fez se conhecer mais, como é esse comportamento aberrante, como a agressividade atua. (P. 3)

Porque aí vai mostrando cada passo, vai mostrando as funções que a gente tem que estar observando. A função que tá(sic) acontecendo, porque que tá(sic) acontecendo e a forma de lidar. Eu achei... Pra (sic) mim, não tenho nada assim que reclamar não. Foi até tranquilo ler. (P.2)

Tais relatos, ao serem comparadas aos resultados do TAl, reiteram a importância da capacitação e a ausência de tais procedimentos na reciclagem dos profissionais da saúde.

\section{MUDANÇAS NO REPERTÓRIO COMPORTAMENTAL DO PACIENTE}

Os participantes não nomearam os procedimentos, porém exemplificaram a intervenção por meio do reforçamento de comportamentos incompatíveis (DRI) que concorriam com o comportamento aberrante. Os participantes P.4 e P.5 descreveram procedimentos topograficamente incompatíveis com o comportamento de se bater/morder, como utilizar as duas mãos em um brinquedo ou cantar/bater as mãos contingente à atenção social. Relataram que os comportamentos aberrantes diminuíram a frequência, sugerindo que os comportamentos incompatíveis foram reforçados. Não foi solicitado no primeiro encontro aos participantes que aplicassem os procedimentos ensinados pelo material, o que sugere generalização desses comportamentos. Na Tabela 5, constam as descrições de intervenções realizadas por meio do procedimento de DRA e de DRI que os participantes relataram:

Porque agora eu consigo, pelo menos um tempo da terapia, fazer com que ele não se bata, porque antes era meia hora, em meia hora ele dando murro. (P.5)

Melhorou, eu conto que melhorou um pouco porque no momento ele não vai se morder. (P.4)

Os relatos verbais de P.4 e P.5 evidenciam melhora no aproveitamento do tempo em terapia com seus pacientes, realizando as atividades, com uma taxa menor de autoagressão. O participante P.6 relatou que atualmente propõe atividade incompatível ao paciente, enquanto este forçava o vômito, utilizando as mãos, passou a solicitar atividades como bater palmas e cantar. Essa intervenção pode ser compreendida como um procedimento de DRI, por ser topograficamente incompatível com o comportamento de enfiar a mão dentro da boca com a finalidade de forçar o vômito, e cuja consequência era atenção. A atenção passou a ser provida por atividades prazerosas como bater palmas e cantar junto com P.6.

Ao serem questionados se conseguiram intervir com 0 paciente da forma como o material havia ensinado, P.3 exemplificou:

A gente tenta fazer bastante coisa né, pra (sic) criança 


\section{3" INTERACÃO EM ES PSICOLOGIA}

interagir, como brinquedos para ele não ficar agressivo. Como eu sou terapeuta ocupacional, eu trabalho bastante com brinquedos para estimular [...] tenta fazer o movimento para controlar a coordenação fina. Daí a gente fala: [nome do paciente], vamos guardar o brinquedo na caixa, ele vai jogando, vai guardando na caixa. (P.3)

Eu consegui agir, mas não adiantou. Eu distraí ele com ... porque o (nome do paciente), ele se bate com a mão, e eu dei um brinquedo, um computador para ele, que tinha o tempo todo que tá (sic) usando a mão. Mas em um minuto de distração, ele se batia de novo. (P.5)

Tem um paciente que já deu pra (sic) usar. O paciente vai se morder e eu começo a cantar: ah, vamo (sic) bater a mão, que não sei o que... (P.4)

\section{MUDANÇAS NA RELAÇÃO PROFISSIONAL - PACIENTE}

Sobre a condição do paciente, se foi observada alguma mudança, todos responderam que notaram alguma melhora, até P.1, que inicialmente respondeu que não interveio como o material ensina, respondeu no TAl adaptado que o comportamento havia melhorado.

[...] eu acho que confiança do paciente em nós. É.... a gente sempre faz a mesma atividade com o paciente, agora que nós começamos a mudar. Ele chegava na sala, [...] a gente colocava o grampo na cadeira. [...] sessão passada, a gente já deixou ele solto. Não sentamos ele na cadeira, para ver como ele ficava. Ele ficou tranquilo. [...] então eu acho que o tempo, né, faz o paciente confiar, a gente saber lidar com o paciente. (P.1)

Pode-se sugerir que P.1 não aprendeu os procedimentos de intervenção ensinados de modo completo, por não relatar nenhuma tentativa de realizá-los, porém aprendeu a observar o comportamento apresentado pelos seus pacientes de forma mais objetiva, observando as consequências dos comportamentos, identificando que, ao ser retirada a consequência que mantinha o comportamento aberrante, ele diminui sua frequência.

\section{SUGESTÕES PARA MELHORIAS NO MATERIAL E VALIDADE SOCIAL}

Os participantes sugeriram uma correção menos detalhada (P.1) e um material mais compacto (P.3 e P.4).

[...] $\mathrm{Na}$ hora de corrigir, acho que você não precisava colocar tão detalhado. (P.1)

O material podia ser um pouco menor. (P.3)

É, eu só assustei com o tamanho do material, deve ter assustado outras pessoas também. (P.4)

A validade social pode ser demonstrada por meio da análise da significância social dos objetivos, a aceitabilidade social dos procedimentos de intervenção e a importância social dos efeitos (Wolf, 1978). Os participantes emitiram opiniões favoráveis quanto ao material, sinalizando que o objetivo da validação social foi alcançado.

O que eu achei mais interessante? É, essas, tipo a tarefinha (sic), os exercícios. Os exercícios que fez assim, fez se conhecer mais (sic), como é esse comportamento aberrante, como a agressividade atua, tipo se arranhar, sabe? Foi muito legal, muito bom mesmo. Vocês estão indo no caminho certo. (P.3)

Sim, tá (sic) bem fácil de entender, eu gostei. (P.4)

Foi boa, muito boa. Porque quando ele apresentava o comportamento eu sempre, principalmente o que estimula vômito, eu sempre deixava ele mais de lado, esperava pra (sic) ver se ele acalmava, porque era falar pra (sic) ele que ele ia pra (sic) fisioterapia, da sala de aula que eu pegava ele até lá ele ia estimulando vômito, aí ficava a terapia inteira então daí eu soube né, como lidar com isso. Antes eu deixava ele de lado, esperava ver se ele acalmava pra (sic) eu conseguir aplicar a Fisioterapia em si. (P.6)

Com a aplicação dos procedimentos ensinados, pode-se considerar, a partir de seus relatos, que os participantes conseguiram realizar as atividades propostas durante seus atendimentos com seus pacientes, observando mais atentamente o comportamento deles, e intervindo para reduzir a frequência do comportamento aberrante. Pode-se concluir que o material elaborado foi validado socialmente pelos participantes do estudo. 0 nível 1 da validade social pode ser exemplificado pela boa aceitabilidade das instituições a participar do estudo, fazendo a intermediação dos funcionários, que também relataram ser um estudo relevante. Os participantes também afirmaram que era um procedimento de intervenção válido, o que implica no nível 2 da validade social de Wolf (1978). Um total de $80,67 \%$ avaliou o material como acima da média; pode-se afirmar, então, que eles ficaram satisfeitos em participar do estudo. Durante as entrevistas, os participantes também indicaram satisfação com o material e em participar do estudo, exemplificaram procedimentos e elogiaram o material. Isso pode demonstrar a validade do estudo e do material desenvolvido. Apesar da validação social não medir a aprendizagem dos participantes, estes relataram alteração em seus comportamentos e tentativas de intervenção em relação aos comportamentos aberrantes de seus pacientes, o que implica em aprendizagem. Embora seja impossível, neste estudo, 


\section{HE' INTERACÃO EM 2. PSICOLOGIA}

quantificar o quanto aprenderam, pode-se afirmar que os participantes aprenderam com o material.

Assim, o material obteve boa avaliação quanto à sua eficácia e efetividade, com relatos de melhoras dos pacientes dos participantes atendidos e com descrição dos procedimentos ensinados pelo material didático.

\section{CONSIDERAÇÕES FINAIS}

Finalizando a discussão dos dados expostos, algumas considerações se fazem relevantes. Em primeiro lugar, os participantes acertaram mais de $80 \%$ das questões do livro, fato que pode ter sido influenciado pela disponibilidade do gabarito imediatamente após os exercícios, para que eles mesmos os corrigissem. 0 gabarito tem o objetivo de sanar dúvidas, oferecer feedback imediato e corrigir possíveis erros de entendimento dos conceitos e/ou exercícios. No entanto, é possível que os participantes tenham lido o gabarito antes de responder as questões, o que poderia implicar no alto número de questões corretas. Vale ainda considerar que o planejamento dos exercícios considerando os princípios dos pequenos passos, feedback imediato, autoavaliação (Botomé, 1970), se bem executado, levaria a um resultado tão bom quanto o encontrado.

A Segunda Unidade (Análise Funcional) é a unidade com mais exercícios dissertativos, porém com $80 \%$ de acertos. Tal índice de acertos, menor que as demais unidades, pode ser atribuída à natureza dos exercícios propostos. Esses exercícios consistiam em realizar observações sistemáticas dos comportamentos, incluindo antecedentes e consequentes, e era solicitado que selecionassem uma alternativa com uma possível função para comportamentos descritos. Tal índice de acertos sugere que sua realização está mais difícil, considerando o público-alvo da pesquisa, demandando uma revisão da unidade.

No entanto, três participantes exemplificaram procedimentos de DRA ou DRI, um indicativo de aprendizado do procedimento de Análise Funcional e dos procedimentos de reforçamento diferencial trabalhados. Considerando a Terceira Unidade, que teve o maior número de acertos, podese inferir que tenha sido bem estruturado, considerando os princípios da programação de ensino. Contudo, assim como na Primeira Unidade, não se pode descartar a possibilidade de que a presença dos gabaritos tenha influenciado no número de acertos. Assim, como preconizado pelos princípios da Programação de Ensino, o material deve estar em constante avaliação, e com os dados obtidos, pode ser necessário rever o nível de dificuldade das unidades que apresentaram uma maior taxa de erros.

Avaliando o resultado das entrevistas em conjunto com o
TAl, foi possível observar que os participantes aplicaram os procedimentos ensinados pelo material, sem a solicitação da pesquisadora. 0 material tem boa aplicabilidade pelo fato de os participantes terem realizado sem nenhuma instrução adicional. No estudo de Hamada (2014) foi realizada uma capacitação de pais de pessoas com deficiência intelectual, com relatos que sinalizam aprendizado e generalização, pois os pais aplicaram os procedimentos ensinados. Estudos com finalidade de ensino de procedimentos analíticocomportamentais aos cuidadores/profissionais/professores podem trazer diversos benefícios para as pessoas com deficiência intelectual e suas famílias, por possibilitarem uma melhor qualidade de vida, aumentando a saúde e a educação desses, e por possibilitarem que os profissionais executem suas competências durante os atendimentos/aulas. Os profissionais participantes relataram melhora no comportamento de seus pacientes, o que pode estar relacionado com a mudança comportamental do próprio participante. A capacitação de pais, profissionais e professores possibilita um melhor ambiente de interação e aprendizado para a criança com deficiência intelectual.

Concluindo, para futuras pesquisas, sugere-se capacitação utilizando o material didático desenvolvido, realização de medição da aprendizagem dos participantes e observação das melhorias no repertório comportamental dos pacientes desses participantes, com a realização de pré e pós-testes e averiguação da aprendizagem, por meio da aquisição de novas competências comportamentais. Além de modificações no próprio material, possibilitando a diminuição do número de erros na Segunda Unidade, assim como melhor controle de variáveis para estudo do material, considerando Primeira e Terceira Unidades, o que provavelmente implicará em aperfeiçoar o material em relação à programação de ensino (Botomé, 1970; Keller, 1968; Skinner,1968/1972).

O objetivo do desenvolvimento desse material, utilizado no presente estudo, foi de preencher a lacuna da falta de materiais, capacitações e recursos para instrumentalizar profissionais da saúde no manejo dos comportamentos aberrantes, haja vista que o conhecimento produzido pela Análise do Comportamento deve ser compartilhado com quem dele necessita. Com a análise das entrevistas e da validação social, é possível afirmar que, dentro desse contexto, é algo novo que acrescenta conhecimentos para os profissionais, alcançando o objetivo social pretendido.

\section{DECLARAÇÃO DA CONTRIBUIÇÃO DOS AUTORES}

T.Y e S.F. contribuíram para a conceitualização, investigação e visualização do artigo; T.Y fez a coleta de dados e a redação inicial do artigo (rascunho) e S.F. é a 


\section{n* INTERACÃO EM 2. PSICOLOGIA}

responsável pela redação final (revisão e edição).

\section{DECLARAÇÃO DE CONFLITOS DE INTERESSE}

Os autores declaram que não há conflitos de interesse no manuscrito submetido.

\section{REFERÊNCIAS}

Ausec, I. C. (2013). Capacitação comportamental informatizada para professores universitários: Inclusão no ensino superior. Dissertação de mestrado, Universidade Estadual de Londrina, Londrina, PR.

Ausec, I. C. O., \& Fornazari, S. A. (2014). Formação docente no ensino superior: capacitação comportamental para inclusão de estudantes com necessidades educacionais especiais. São Carlos: Marquezine \& Manzini, ABPEE.

Bassetto, V. H. (2015). Software "ensino" para capacitação em Análise do comportamento: avaliação da eficiência entre duas versões. Dissertação de mestrado, Universidade Estadual de Londrina, Londrina, PR.

Botomé, S. P. (1970). Princípios básicos para programação de ensino. Não publicado.

Carlomagno M. C., \& Rocha, L. C. (2016). Como criar e classificar categorias para fazer análise de conteúdo: Uma questão metodológica. Revista Eletrônica em Ciência Política, 7(1), 173-188.

Conyers, C., Miltenberger, R., Romaniuk, C., Kopp, B., \& Himle, M. (2003) Evaluation of DRO schedules to reduce disruptive behavior in a preschool classroom. Child \& Family Behavior Therapy, 25(3), 1-6.

Couto, C. M. (2009). Educação a distância e sistema personalizado de ensino: avaliação de um curso utilizando o sistema Capsi. Dissertação de mestrado, Pontifícia Universidade Católica de São Paulo, São Paulo. Recuperado de https://tede2.pucsp.br/handle/handle/ 16868

Eyberg, S. (1993). Consumer satisfaction measures for assessing parent training programs. Em L. Vandecreek; S. Knapp; S. T. L. Jackson (Orgs.). Innovations in clinical practice: A source book (Vol. 17). Sarasota: Professional Resource Press.

Eyre, H. L. (2007). Keller's Personalized System of Instruction: Was it a Fleeting Fancy or is there a Revival on the Horizon? The Behavior Analyst Today, 8(3), 317-324. http:// dx.doi.org/10.1037/h0100623
Eyre, H. L., Crone-Todd, D. E., Jones, J. R., Hutchens, S. A., \& Pear, J. J. (2005). Using CAPSI to increase student engagement in general psychology. Delta Education Journal, 1(25), 33-40.

Fornazari, S.A (2000). Redução de comportamentos inadequados em portadores de deficiência mental, no treino, para o trabalho. Dissertação de mestrado, Universidade Federal de São Carlos, São Carlos.

Fornazari S. A (2005). Comportamentos inadequados e produtividade em pessoas com deficiência mental ou múltipla em ambiente educacional. Tese de doutorado, Universidade Estadual Paulista Júlio de Mesquita Filho, Araraquara.

Fornazari, S. A. (2011). Software "ENSINO" (versão 1.5.5) [Programa de computador]. Universidade Estadual de Londrina, Londrina, PR.

Fornazari, S.A., Hamada, R. A., Rizardi, C. M., Inácio, F. F., Devides, M. B. C., Salviati, M. R., \& Dias, M. F. (2014). Análise do comportamento aplicada às pessoas com necessidades educacionais especiais: programa de capacitação para profissionais da saúde. In V. B. Haydu, Fornazari, S. A., \& Estanislau, C. R. (Orgs.). Psicologia e Análise do Comportamento: Conceituações e Aplicações à Educação, Organizações, Saúde e Clínica (pp. 115-136). Londrina: UEL.

Fornazari, S. A., Kienen, N., Tadayozzi, D. S., Ribeiro, G. D., \& Rossetto, P. B. (2012). Capacitação de professores em análise do comportamento por meio de programa educativo informatizado. Psicologia da Educação, 35, 2452.

Friman, P. C., Barnard, J. D., Altman, K., \& Wolf, M. M. (1986). Parent and teacher use of DRO and DRI to reduce aggressive behavior. Analysis and Intervention in Developmental Disabilities, 6(4), 319-330. https://doi.org/ 10.1016/S0270-4684(86)80012-X

Gomes, C., \& Barbosa, A. J. G. (2006). Inclusão escolar do portador de paralisia cerebral: atitudes dos professores do ensino fundamental. Revista Brasileira de Educação Especial, 12(1), 85-100.

Hamada, R. A. (2014). Comportamentos Aberrantes: Revisão de estudos sobre o tema e elaboração de material didático para pais. Dissertação de mestrado, Universidade Estadual de Londrina, Londrina, PR.

Höher, S.P., \& Dotto L. W. A. (2006). A transmissão do diagnóstico e de orientações pais de crianças com necessidades especiais: a questão da formação profissional. Estudos de Psicologia, 23(2), 113-125

Keller, F. S. (1968). "Good-bye teacher...". Journal of Applied Behavior, 1(1), 79-89. https://doi.org/10.1901/jaba. 1968.1-79 


\section{W'INTERACÃO EM ET. PSICOLOGIA}

Kienen, N (2008). Classes de Comportamentos Profissionais do Psicólogo para Intervir, por Meio de Ensino, Sobre Fenômenos e Processos Psicológicos, Derivadas a Partir das Diretrizes Curriculares, da Formação Desse Profissional e de um Procedimento de Decomposição de Comportamentos Complexos. Tese de doutorado, Universidade Federal de Santa Catarina, Santa Catarina. SC. Recuperado de https://repositorio.ufsc.br/xmlui/ handle/123456789/92016?show=full

Leonardo, N. S. T., Bray, C. T., \& Rossato, S. P. M. (2009). Inclusão escolar: um estudo acerca da implantação da proposta em escolas de ensino básico. Revista Brasileira de Educação Especial, 15(2), 289-306. http://dx.doi.org/ $10.1590 /$ S1413-65382009000200008

Lennox, D. B., Miltenberger, R. G., Sprengler, P. M., \& Erfanian, N. (1988). Decelerative treatment practices with persons who have mental retardation: A review of five years of the literature. American Journal on Mental Retardation, 92(6), 492-501.

Liddon, C. J., Kelley, M. E., \& Podlesnik, C. A. (2017) An animal model of differential reinforcement of alternative behavior. Learning and Motivation, 58, 48-58. https:// doi.org/10.1016/j.Imot.2017.04.001

Maciel, M. R. C. (2000). Portadores de deficiência: A questão da inclusão social. São Paulo em Perspectiva, 14(2), 5156. http://dx.doi.org/10.1590/S0102-

\section{8}

Martins, E. V. (2013). Aplicação e avaliação de duas capacitações informatizadas para pais de crianças com problemas de comportamento. Dissertação de mestrado, Universidade Estadual de Londrina, Londrina, PR.

Mello, H. C. M. (2012). Avaliação de um programa informatizado de capacitação para mães de crianças com necessidades especiais. Dissertação de mestrado, Universidade Estadual de Londrina, Londrina, PR.

Mendes, E. G. (2001). Comportamentos aberrantes ou desafiadores? In M. C. Marquezine, M. A. Almeida, \& E. D. O. Tanaka (Orgs.). Perspectivas multidisciplinares em educação especial II (pp. 321-332). Londrina: Eduel.

Miltenberger, R. G. (2001). Behavior modification: Principles and procedures (2 Ed.). Belmont, CA: Wadsworth/ Thomson Learning.
Moreira, M. B., \& Medeiros, C. A. (2007). Princípios básicos de análise do comportamento. Porto Alegre: Artmed.

O’Brien, S., \& Repp, A. (1990). Reinforcement-based redutive procedures: A review of 20 years of their use with persons with severe or profound retardation. Journal os the Association for Persons with Severe Handicaps, 15(3), 148-159. https://doi.org/10.1177/154079699001500307

Petscher, E. S., Rey, C., \& Bailey, J. S. (2009). A review of empirical support for differential reinforcement of alternative behavior. Research in Developmental Disabilites, 30(3), 409-425. https://doi.org/10.1016/j.ridd. 2008.08.008

Piazza, C. C., Moes, D. R., \& Fisher, W. W. (1996). Differential reinforcement of alternative behavior and demand fading in the treatment of escape-maintained destructive behavior. Journal of Applied Behavior Analysis, 29(4), 569572. https://doi.org/10.1901/jaba.1996.29-569

Roane, H. S., Fisher, W. W., Sgro, G. M., Falcomata, T. S., \& Pabico, R. R. (2004). An alternative method of thinning reinforcer delivery during differential reinforcement. Journal of Applied Behavior Analysis, 37(2), 213-8. https:// doi.org/10.1901/jaba.2004.37-213

Skinner, B. F. (1968/1972). Tecnologia de ensino. São Paulo: Editora Pedagógica e Universitária Ltda.

Suárez, A. B. (2000). Reflexiones acerca del uso de los conceptos de eficiencia, eficacia y efetividad en el sector salud. Revista Cubana de Salud Publica, 26(1), 50-56.

Wolf, M. M. (1978). Social validity: The case of subjective measurement or How Applied Behavior Analysis is finding its heart. Journal of Applied Behavior Analysis, 11(2), 203214. https://doi.org/10.1901/jaba.1978.11-203

Yazawa, T. (2015). Comportamentos aberrantes: revisão de procedimentos de intervenção, elaboração e avaliação de material didático para profissionais da saúde. Dissertação de mestrado, Universidade Estadual de Londrina, Londrina, PR.

Data de submissão: 09 de janeiro de 2018 Primeira decisão editorial: 09 de agosto de 2018 Aprovação: 05 de fevereiro de 2019 